\title{
MODELING OF OXYGEN-NEON DOMINATED ACCRETION DISKS IN ULTRACOMPACT X-RAY BINARIES: 4U 1626-67
}

\author{
K. Werner, ${ }^{1}$ T. Nagel, ${ }^{1}$ S. Dreizler ${ }^{2}$ and T. Rauch $^{3,1}$
}

\begin{abstract}
RESUMEN
Presentamos los primeros resultados computacionales de espectros sintéticos de discos de acreción pobres en $\mathrm{H} / \mathrm{He}$ en ultracompactas LMXBs. Nuestro objetivo es la determinación de la composición química de la estrella donadora de muy baja masa que forma el núcleo de lo que fue una enana blanca $\mathrm{C} / \mathrm{O}$. El análisis de abundancias nos permite obtener conclusiones sobre el asentamiento gravitacional en WDs, un importante proceso que afecta a los tiempos de enfriamiento y a los períodos pulsantes de los modos $\mathrm{g}$.
\end{abstract}

\section{ABSTRACT}

We report on first results of computing synthetic spectra from $\mathrm{H} / \mathrm{He}$-poor accretion disks in ultracompact LMXBs. We aim at the determination of the chemical composition of the very low-mass donor star, which is the core of a former $\mathrm{C} / \mathrm{O}$ white dwarf. The abundance analysis allows to draw conclusions on gravitational settling in WDs which is an important process affecting cooling times and pulsational g-mode periods.

\section{Key Words: ACCRETION, ACCRETION DISKS - BINARIES: CLOSE - WHITE DWARFS}

\section{PROPERTIES OF 4U 1626-67}

$4 \mathrm{U} 1626-67$ is a low-mass X-ray binary with an orbital period of $42 \mathrm{~min}$. The accretor is a $7.7 \mathrm{~s} \mathrm{X}$ ray pulsar with a magnetic field strength of $3 \cdot 10^{12} \mathrm{G}$ (Orlandini et al. 1998). The donor is a very low mass degenerate star with $\mathrm{M}=0.02-0.08 \mathrm{M}_{\odot}$ (Chakrabarty 1998). Its progenitor is probably a white dwarf with a $\mathrm{C} / \mathrm{O}$ core. The enhanced neon abundance observed in the accretion disk is the result of chemical fractionation within the white dwarf core (Yungelson et al. 2002). According to Chakrabarty (1998) the accretion disk has an inner radius of $6500 \mathrm{~km}$ (corotation radius) and the outer radius is tidally truncated at $200000 \mathrm{~km}$. The mass-transfer rate amounts to $2 \cdot 10^{-10} \mathrm{M}_{\odot} / \mathrm{yr}$ and the inclination angle is either close to $8^{\circ}$ or $33^{\circ}$. Chandra spectroscopy (Schulz et al. 2001) suggests that the disk's chemical composition is $\mathrm{O}-\mathrm{Ne}$ rich. The observed emission lines from highly ionized $\mathrm{O}$ and $\mathrm{Ne}$ are double peaked and probably stem from the Keplerian rotating accretion disk. Absorption edges in the X-ray spectra point to a $\mathrm{C} / \mathrm{O} \mathrm{WI}$ donor. HST/STIS observations appear to corroborate the $\mathrm{H}-\mathrm{He}$ poor chemistry in the disk. The UV spectra show double peaked emission lines, e.g. from $\mathrm{CIV}$ and $\mathrm{OV}$ but do not show the He II $1640 \AA$ line (Homer et al. 2002). A quantitative spectral analysis of the accretion disk composition would allow the test the idea that the donor

\footnotetext{
${ }^{1}$ IAAT, Cniversität Tübingen, Germany

${ }^{2}$ Universitätssternwarte Göttingen, Germany

${ }^{3}$ Sternwarte Bamberg, Germany
}

is a stripped $\mathrm{C} / \mathrm{O}$ white dwarf. We could also determine abundances of heavier elements $(\mathrm{Ne}, \mathrm{Mg}$ ), which would enable us to quantitatively test theories about gravitational settling of these elements in WD cores. This process is intensively debated because it significantly affects WD cooling times and also g-mode periods in pulsating WDs (e.g. Deloye \& Bildsten 2002).

$4 \mathrm{U} 1626-67$ is not unique. It belongs to a small group of six ultracompact LMXBs $\left(P_{\text {orb }} \lesssim 80 \mathrm{~min}\right.$; see e.g. Juett et al. 2001$)$ with very low mass $\left(\lesssim 0.1 \mathrm{M}_{\odot}\right)$ $\mathrm{H}$-poor donors. The binary separation is of the order 1 light-s (Earth-Moon distance) and mass-transfer is driven by gravitational radiation. The optical emission is dominated by their X-ray heated accretion disk and shows no hydrogen lines. This has been reinforced by recent spectroscopy of three group members (Nelemans et al. 2003).

\section{DISK MODELING}

Modeling is performed with a newly developed non-LTE code (Nagel 2003), that is based on an advanced stellar atmosphere code (Werner et al. 2003). Assuming that the radial disk structure is that of an $\alpha$-disk (Shakura \& Sunyaev 1973), we model the vertical structure of the disk and the emerging spectrum as realistic as possible. For that, we divide the disk into a number of concentric annuli and assume that each annulus radiates as a plane-parallel slab. We solve consistently the radiation transfer equations and the non-LTE rate equations for the atomic populations together with the hydrostatic and energy 


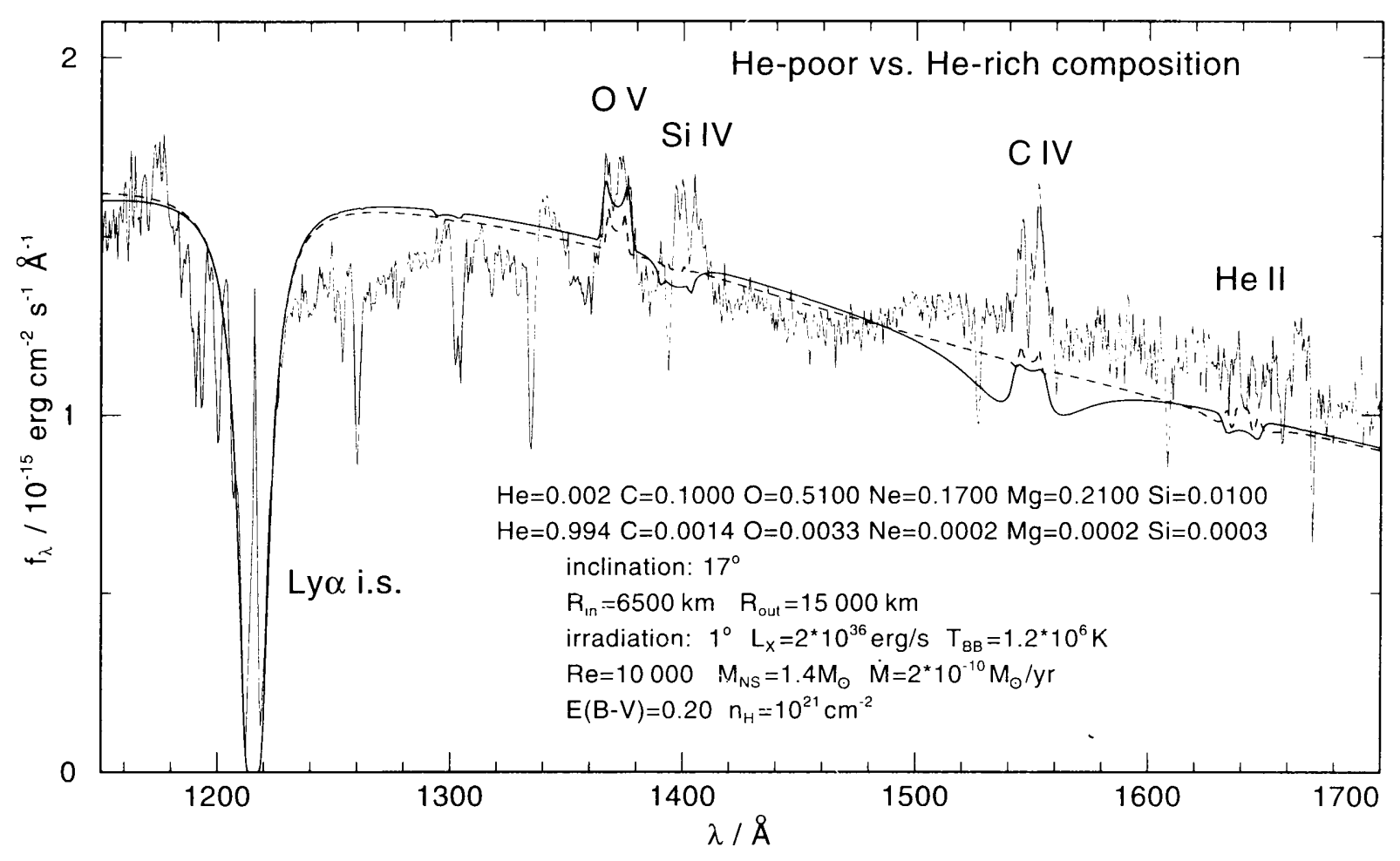

Fig. 1. HST/STIS accretion disk spectrum of 4U 1626-67 and two model spectra, differing by their helium abundance. One is He-poor ( $0.2 \%$ by mass, full line), the other is He-rich (99\%, dashed line). The observed lack of He II 1640 $\mathrm{t}$ obviously is no proof for the He-deficiency of the disk. Note the broad absorption wings of the ('IV resonance line in the He-poor model, which are not observed. This points at a stronger irradiation than assumed.

equations. We can account for full metal line blanketing. This is important because pressure broadening of spectral lines in the dense LMXB disks affects the vertical structure by blanketing and backwarming effects. The kinematic viscosity is parameterized in terms of a Reynolds number (here we set $R e=10000$ which corresponds to $\alpha=0.01-0.1$ ).

For our model of the hot inner disk regions of $4 \mathrm{U}$ 1626-67, where the UV spectrum is formed, we assume $M_{\mathrm{NS}}=1.4 \mathrm{M}_{\odot}$. The adopted disk composition considers the results from X-ray spectroscopy (Schulz et al.2001). We assume a $\mathrm{H}$-free and strongly He-poor disk with high amounts of $\mathrm{O}$ and $\mathrm{Ne}$ (see Fig. 1). For comparison we computed a Hedominated disk with a composition that represents an AM CVn disk chemistry. We also assume that the disk is irradiated by a central source with a blackbody spectrum with $\mathrm{T}=1.2 \cdot 10^{6} \mathrm{~K}$ and a luminosity of $\mathrm{L}=2 \cdot 10^{36} \mathrm{erg} / \mathrm{s}$. We present first results of our attempts to fit the HST/STIS spectrum in Fig. 1. The spectral lines can be modeled qualitatively and the main result is, that a He-rich disk composition cannot be ruled out from this observation. We will perform VLT optical spectroscopy to look for He II $4686 \AA$ and our next step aims at analyzing the $\mathrm{X}$-ray emission line spectra.

This work was supported by DFG (Wo $1312 / 2$ ) and DLR (50 OR 0201). We thank Lee Homer for sending us his reduced HST/STIS spectrum.

\section{REFERENCES}

Chakrabarty, D. 1998, ApJ. 492. 342

Deloye, C. J., Bildsten, L. 2002, ApJ. 580. 107

Homer, L., Anderson, S. F., Wachter, S. 200'2. A.J, 1.24. 3348

Juett, A. M., Psaltis, D.. Chakrabarty. D. 2001. IpJ. 560, L59

Nagel, T. 2003, Dissertation. Lniversity of Tübingen

Nelemans, G., et al. 2003, MNRAS. in press

Orlandini, M., et al. 1998, ApJ, 500, L163

Schulz, N. S., et al. 2001, ApJ, 563. 941

Shakura, N. I., Sunyaev, R. A. 1973, A.'A. 24. 337

Werner, K., et al. 2003, in Stellar ttmosphere Modeling. eds. I. Hubeny, D. Mihalas, K. Worner. ASP Conf. Ser. 288,31

Yungelson, L. R., Nelemanis, G., van den Heuvel. E. P J. 2002, A\&A. 388, 546

Stefan Dreizler: Univ.-Sternwarte, D-73083 Göttingen. Germany (dreirler.astrophysik.uni-goettingen.de). Thorsten Nagel, Thomas Rauch, Klaus Werner, Institut für Astrorromie und Astrophysik, Universit ät Tübingenl.

Sand 1, D-72076 Tübingen, Germany (nagel, rauch, werner(iast ro.uni-tuebingen.de). 